\title{
South African healthcare provider perspectives on transitioning adolescents into adult HIV care
} \author{
L-G Bekker, ${ }^{1} \mathrm{MB} \mathrm{ChB}, \mathrm{DTMH}, \mathrm{DCH}, \mathrm{PhD}$ \\ ${ }^{1}$ Desmond Tutu HIV Centre, Faculty of Health Sciences, University of Cape Town, South Africa \\ ${ }^{2}$ School of Medicine, Stanford University, Stanford, CA, USA \\ ${ }^{3}$ School of Medicine, Harvard University, Boston, MA, USA \\ ${ }^{4}$ Metro-District Health Services, Gugulethu, Cape Town, South Africa
}

T H Kung, ${ }^{1,2}$ BA; M L Wallace, ${ }^{1} \mathrm{MSc}$, PhD; K L Snyder, ${ }^{1} \mathrm{MSc}$; V K Robson, ${ }^{1,3}$ BS; T S Mabud ${ }^{2}$ MSc; C D Kalombo, ${ }^{1,4}$ MB ChB;

Corresponding author: TH Kung (tkung@stanford.edu)

\begin{abstract}
Background. The first generation of South African (SA) children perinatally infected with HIV is entering adulthood, and there is now a pressing need for systematised transfer of these patients from paediatric to adult care.

Objectives. Previous research has investigated the HIV healthcare transition in North America and Europe, yet none has been conducted in SA. Our study is the first to describe the perspectives of healthcare providers overseeing the transition in resource-limited settings.

Methods. We approached healthcare providers working in government paediatric HIV clinics and hospitals in the Western Cape Province, SA. Seven physicians and counsellors in adolescent/paediatric care, representing five clinics, were interviewed, and 43 completed a written survey. Interviews addressed the current state of the transition, barriers and facilitators, and model components. Interviews were assessed for major themes using framework analysis, while logistic regression was applied to survey responses to identify associations with measured covariates. Results. Analysis of interview transcripts revealed several overarching perspectives that were corroborated by survey responses. One barrier identified was the healthcare providers' difficulty in letting go of their relationships with the adolescent patients. Since healthcare providers regarded their patients as particularly vulnerable, they felt a strong and protective attachment towards them. A second barrier identified was a lack of structure and effective communication between adult and paediatric providers; accordingly, healthcare providers feared that they were transferring their adolescents unprepared, to a judgemental, depersonalised and overburdened environment. All interviewees and a majority of survey respondents $(>80 \%)$ agreed that the formation of adolescent support groups in adult care clinics as well as a later transition age would improve the transition process.

Conclusion. This study highlights the need for a systematic healthcare transition for HIV-positive adolescents cared for in the Western Cape, while acknowledging the limitations of the current healthcare infrastructure. Several feasible recommendations have been identified, including forming support groups and greater involvement of adolescent healthcare providers to facilitate the transition.
\end{abstract}

S Afr Med J 2016;106(8):804-808. DOI:10.7196/SAMJ.2016.v106i8.10496

Until recently, most South African (SA) children infected perinatally with HIV did not survive until adulthood. Currently, with early provision of freely available highly active antiretroviral therapy (HAART), these children are expected to live long, healthy lives. ${ }^{[1]}$ SA healthcare providers are presently facing an unprecedented challenge. The first generation of SA children perinatally infected with HIV is entering adulthood, and there is now a pressing need for systematised transfer of these patients from paediatric to adult care, known as healthcare transition.

In North America and Europe, adolescents diagnosed with chronic medical conditions typically complete their healthcare transition between the ages of 18 and 24 in systematic and structured processes in some programmes. ${ }^{[2]}$ Healthcare transition for adolescents living with HIV is recommended but not widely practised even in these developed settings. Poorly conducted healthcare transitions can lead to treatment non-adherence and loss to follow-up, with medical, social and educational repercussions. ${ }^{[2,3]}$ Barriers to an effective transition for adolescents living with HIV include developmental delays, mental health issues, stigma and lack of a supportive social network or home environment..$^{[4,5]}$

Several healthcare transition models have been developed in high-resource setting ${ }^{[6]}$ but are not directly translatable to SA owing to cost, workforce burden, and cultural and contextual differences. Additional patient-specific barriers faced by SA adolescents living with HIV include high levels of stigma, late presentation of illness, and inadequate home and family support. ${ }^{[2,7,8]}$ At the community level, adolescents face unemployment, poverty and crime. ${ }^{[8]}$ At the national level, patients and providers face a health infrastructure already strained to meet the enormous scale of SA's antiretroviral therapy (ART) programme. Adolescents often leave youth-friendly care to enter adult clinics characterised by limited space, time, staffing and other resources.

\section{Objectives}

Little research has been conducted on the HIV transition in resourcelimited settings, and few studies worldwide have looked at the point of view of the healthcare provider overseeing the transition. ${ }^{[2,4]}$ Our study is the first to describe the perspectives of SA providers concerning the transition process. For a formalised healthcare transition approach to be applied successfully, the models proposed in previous studies would need to be perceived as acceptable and feasible by implementing healthcare providers. These data are part of a larger formative study examining attitudes towards and experiences of this transition process in adolescent patients and healthcare providers. This study qualitatively and quantitatively investigated attitudes and experiences from a sample of healthcare providers in paediatric, adolescent and adult HIV care clinics in the Western Cape Province, SA. 


\section{Methods}

\section{Ethics statement}

The University of Cape Town Human Research Ethics Committee approved this study (Ref. No. 167/2013). Permission was also granted by the Western Cape Department of Health (Ref. No. RP 054/2013). Informed consent was obtained from all study participants.

\section{Data collection}

Using convenience sampling, we approached healthcare providers working in five government HIV care clinics in the Western Cape to participate in an interview study evaluating their perspectives on the adolescent healthcare transition. We recruited seven healthcare providers from these clinics through email or in-person invitations. In four of the five clinics represented, teenage patients transfer from paediatric to adolescent care, in which youth remain at the same clinic but see providers on a separate 'adolescent day' of the week. Table 1 details interviewee specialties, clinical environment and patient information.

Semistructured interviews lasting $\sim 40$ minutes were conducted in English and audio-recorded in a private space with only the healthcare provider and facilitator (one of two research team members) present. The interview guide was developed in line with HIV healthcare transition literature, ${ }^{[2,4,6]}$ addressing: the current transition process, adolescent v. adult clinics, transition barriers, reactions to a formalised transition model, resource availability and interviewee recommendations for improving the local transition process.

Beyond the interview study, 43 additional local HIV healthcare providers participated in a paper or electronic survey (response rate 52\%). Through convenience sampling, electronic surveys were emailed to local HIV healthcare providers (physicians, nurses, counsellors) while paper surveys were handed to providers practising at clinics affiliated to the Desmond Tutu HIV Foundation. Survey questions addressed barriers to the transition process much like the interviews, and additionally assessed provider perspectives on the helpfulness and feasibility of several proposed interventions. Survey items were presented as a series of statements to which the healthcare providers responded with a four-point Likert scale, from 'Strongly disagree' to 'Strongly agree' (a neutral value was not used, to prevent participant confusion). Interviewees and survey respondents were not compensated for participation.

\section{Data analysis}

Interviews were transcribed verbatim and analysed with the Framework Analysis methodology, developed by the UK National Centre for Social Research. ${ }^{[9]}$ This approach, which has been used extensively for applied qualitative research, organises data and facilitates case analysis according to key themes, concepts and emergent categories.
We used a transcript coding scheme following prior qualitative studies of the HIV healthcare transition model..$^{[4,6,10]}$ Each transcript was coded twice to improve precision. The same transcript was then codified independently by at least one other investigator, and reconciled with the original code to ensure inter-rater reliability. ${ }^{[11,12]}$ We then applied multiple matrices to identify similarities, contrasts and interrelations between the perspectives of the interviewees by specialty, clinical environment, transition destination of patients and patient mode of infection. ${ }^{[12]}$

We organised and tabulated survey responses in an electronic database. We then applied univariate logistic regression to survey responses using R (version 3.1.2; R Development Core Team, New Zealand) to assess associations between provider type (clinician, nurse or counsellor) and the level of agreement to survey items. A Bonferroni correction was applied to account for multiple comparisons.

\section{Results}

\section{Lack of a structured healthcare transition}

All seven interviewees stated that their current paediatric and adolescent HIV patients are the first to survive into adulthood. The need for transitioning patients has surprised providers, and none of the five clinics represented had a systematised transition process in place. Several interviewees had not yet made their patients aware of the need to transition, and accordingly felt their patients could reasonably expect that they could stay in adolescent care indefinitely.

All interviewees claimed to have struggled in deciding when to transfer their patients out of paediatric/adolescent care, because no guidelines existed for this process at the national level, or in the clinics investigated. Those of the healthcare providers who considered it mentioned several signals they had used to justify starting the transition process, including a patient's: age (ranging from 16 to 25 years across providers), completion of high school, request to move to adult care, and completion of the Xhosa manhood initiation ritual involving male circumcision. Six interviewees stated that pregnancy was another clear indicator that young women adolescents were ready to transfer to adult care, regardless of the female's age.

All seven interviewees stated that adolescent care was over capacity at their respective institutions, and that they were in need of a structured transition model. Healthcare providers believed that adolescent care was over-burdened with an increased number of patients that current infrastructure could not support.

\section{Lack of communication between adolescent and adult care physicians}

Of the five interviewees who needed to transfer adolescent patients to a separate ART clinic in the townships, four agreed that one of the main barriers providers faced in the transition was a lack of

Table 1. Interview participant characteristics

\begin{tabular}{|c|c|c|c|c|}
\hline Patient population & Position & $\begin{array}{l}\text { Clinical environment of } \\
\text { adolescent patients }\end{array}$ & $\begin{array}{l}\text { Primary mode of } \\
\text { patient infection }\end{array}$ & $\begin{array}{l}\text { Transition destination } \\
\text { for adult ART care }\end{array}$ \\
\hline Adolescent & Medical officer & Tertiary care government hospital & Vertical & Move to township \\
\hline Adolescent & Medical officer & Tertiary care government hospital & Vertical & Move to township \\
\hline Paediatric and adolescent & Senior paediatrician & Tertiary care government hospital & Vertical & Move to township \\
\hline Adolescent & Youth counsellor & Township ART clinic & Vertical & Next door \\
\hline Adult and adolescent & Medical officer & Township ART clinic & Vertical & Next door \\
\hline Adolescent & Youth counsellor & Township ART clinic & Horizontal & Separate township \\
\hline Adolescent & Medical officer & Township ART clinic & Vertical & Separate township \\
\hline
\end{tabular}


communication between paediatric and adult providers. These paediatric providers claimed that they were unable to refer their patients to a specific adult physician - only to the adult clinic as a whole. There was no handover between paediatric and adult physicians; after transitioning, patients therefore received treatment from physicians who had little to no prior knowledge of patient history or conditions. Only 55\% of survey respondents agreed that there was effective communication between adult and paediatric providers during the transition, and $71 \%$ of respondents believed that adolescents found adult care to be scary or unfriendly (Table 2). All seven adolescent healthcare providers expressed fears about sending their patients to adult care unprepared, with no one on the receiving end to help the youth navigate the new system.

\section{Lack of quality adult care}

All seven interviewees acknowledged that paediatric health services in the Western Cape are among the most comprehensive available within the SA public healthcare system. Paediatric providers were proud that their clinics offered holistic care: mental health services, access to counselling, home visits for missed appointments, patient follow-up, family planning services integration, and other linkages to care. A majority $(71 \%)$ of surveyed providers agreed that patients would find it easier to adhere to medications in adolescent care over adult care. Furthermore, interviewees stated that the parents or guardians of young patients recognised that their children received superior services at the adolescent clinic. Paediatric providers recounted numerous occasions when they were pressured by patients' parents to keep their children in adolescent care.

Youth healthcare providers aimed to follow up with their own patients at each visit, and they recognised their privilege in forming long-term relationships with adolescents. Interviewees described how, within each appointment, the physicians, counsellors and nurses in paediatric care could spend significantly more time with each patient compared with in adult clinics. Providers stated that their youth clinics served roughly 500 patients, while the local adult HIV clinics served as many as ten times this number of adults. All seven interviewees explained that they were strongly attached to their vulnerable patients, the majority of whom were orphans or had experienced significant trauma. The providers experienced difficulty letting go of these relationships, believing that they had the most resources and attention to enable patients to be healthy and successful. Interviewees referred to their adolescent patients as their 'babies' and expressed reluctance in transferring them out to what they perceived to be relatively depersonalised, overburdened and unwelcoming adult services.

\section{Mental health problems and neurocognitive delays}

All seven adolescent healthcare providers stressed that their perinatally infected patients faced delayed puberty, intellectual impairments and, critically, mental health issues. Adolescent providers believed that adult providers, lacking intimate knowledge of incoming adolescent patients, would not be able to properly address these additional health issues. A majority of survey respondents (88\%) agreed that mental health problems could interfere with the transition process and this inadequate mental health support could present a challenge.

\section{Patient attachment to adolescent healthcare providers and facilities}

In all five sites interviewed, adolescents had 'chill rooms', which were often equipped with recreational items, e.g. a pool table, free lunch and a support group. Providers believed that patients viewed their clinic visits as an 'outing', and they frequently encountered patients spending time at the clinic even when they were not seeking healthcare.

All seven interviewed participants and $80 \%$ of survey respondents claimed that the transitioning adolescents had difficulties in letting go of their adolescent caregivers. Providers articulated that most patients had attended the clinic for their entire lifetime - many patients even received regular care at the clinic prior to the ART roll-out in 2003. Commonly, patients were extremely ill when they first entered the clinic and made a dramatic recovery in paediatric care. Interviewees reported that the patients grew up in the clinic and

Table 2. Summary of survey responses: provider perspectives on barriers and facilitators to healthcare transition

\begin{tabular}{ll}
\hline & $\begin{array}{l}\text { Agree or } \\
\text { strongly agree, } \\
\%(N=\mathbf{4 3})\end{array}$ \\
\hline Potential barrier or facilitator to transition & 34 \\
Attending the adult clinic requires patients to spend more money on transportation & 63 \\
Patients find it easier to adhere to the medication regimen after transitioning to adult care & 29 \\
Crime and/or drug abuse in the community may interfere with adolescents' transition process & 80 \\
Patients have supportive friends and/or family who can help them in the transition from adolescent to adult care & 63 \\
Mental health problems such as depression or anxiety often make it difficult for a patient to move to the adult clinic & 88 \\
Adolescents have no issues with trusting a new provider & 22 \\
The neurocognitive delay associated with perinatally infected HIV-positive adolescents interferes with the transition process & 83 \\
The stunted growth and delayed puberty in many perinatally infected adolescents is a barrier to the healthcare transition & 60 \\
Stigma and discrimination are issues that affect the adolescent's transition to the adult clinic & 78 \\
Adolescents have sufficient education about HIV infection and medications & 51 \\
There is effective communication between paediatric and adult providers during the transition process & 55 \\
Adolescents have difficulty letting go of their relationship with the adolescent provider & 80 \\
Adolescents perceive adult care to be scary or unfriendly & 71 \\
Adolescents have the independence and autonomy to transition to adult care & 55
\end{tabular}


built strong relationships with the adolescent caregivers who nursed them back to health. Providers recognised that special attention and continuity of care was often not possible in adult care.

\section{Reciprocal stigmatisation}

Six of seven interviewees stated that adolescents feared disclosure of their HIV status in adult HIV clinics. This concern about confidentiality was even more pronounced for patients moving to primary healthcare clinics in townships. Interviewees suspected that adolescent patients would feel exposed when entering communitybased adult HIV clinics filled with relatives, neighbours and other community members.

Newly transitioned adolescent patients appear noticeably younger than average adult HIV patients, and healthcare providers stated adolescents feared stigma from unwelcoming adult patients and staff. Since most patients in adult care were infected as adults, there was anxiety that many might assume the adolescents were behaviourally infected, with inherent associated stigma; interviewees reported that adolescent HIV patients were often viewed as promiscuous and irresponsible. Paediatric providers further emphasised that adult staff were not trained to work with younger people, and were sometimes judgemental concerning teenage decision-making and sexual behaviours.

Ironically, interviewees also believed that adolescents stigmatised adult patients. They stated that adolescents perceived adult patients to be old and sick with TB, very unlike their HIV-positive peers. Additionally, interviewees discussed perceptions of blame in HIV transmission. Providers suspected that the perinatally infected adolescents perceived that older patients acquired HIV through their own actions, while adolescents did not attribute the same fault towards their own perinatally infected cohort. Paediatric providers perceived that perinatally infected patients saw their identity as wholly separate from adult HIV patients, and recognised an immense solidarity among adolescents that could be lost in transition.

\section{Recommendations}

Although interviewees and survey respondents believed that implementing the transition models published in resource-rich countries would facilitate an ideal transition, ${ }^{[6]}$ they were concerned about feasibility in the SA setting, in particular: comprehensive follow-up once the patient has transferred out is unlikely; the engagement of the adult doctor in processes such as meeting the patients in the adolescent clinic is unrealistic; and they currently lack resources for hiring staff such as social workers to accompany adolescents on their initial adult clinic visits.

Survey respondents largely agreed the most helpful (100\%) and feasible (88\%) proposed intervention would be to create support groups for transition-age adolescents (Table 3), which was corroborated by interviewees. This is interesting in the light of recently developed community-based adherence clubs being tried in adult care in the Western Cape. Understanding how these two concepts may be brought together for better adolescent healthcare transition is worthy of testing. Interviewees provided practical and contextually appropriate recommendations to improve the SA healthcare transition (Table 4).

\section{Discussion}

We provide here the first assessment of SA HIV paediatric/adolescent physicians and counsellors' perceptions of the healthcare transition process. In keeping with studies in other contexts, a barrier to the transition process was the healthcare providers' difficulty in letting go of relationships with their adolescent patients. ${ }^{[4,13]}$ Healthcare providers felt a strong, protective attachment towards their adolescent patients, whom they considered vulnerable. The adolescent clinic physicians and counsellors were proud of the care they provided in the adolescent clinic and recognised that the patients were reciprocally attached to the staff and space. Since current transition lacked structure and effective communication between providers on both sides, paediatric providers feared they were transferring their adolescent patients unprepared into a judgemental, depersonalised and overburdened environment.

Attachment has been widely researched in various patientcaregiver relationships; for example, where relatives provided care, ${ }^{[14]}$ or in relationships between providers and patients with chronic medical conditions. ${ }^{[15]}$ The attachment that healthcare providers experience towards their patients is relatively understudied, but has materialised briefly in literature regarding healthcare transitions..$^{[4,13]}$ The protectiveness healthcare providers feel towards adolescent patients can be harnessed to improve the healthcare transition. While

Table 3. Summary of survey responses: provider perspectives on helpfulness and feasibility of proposed interventions

\begin{tabular}{lll}
\hline & $\begin{array}{l}\text { Helpful: agree } \\
\text { or strongly } \\
\text { agree, \% }\end{array}$ & $\begin{array}{l}\text { Feasible: agree } \\
\text { or strongly } \\
\text { agree, \% }\end{array}$ \\
\hline $\begin{array}{l}\text { Developing written guidelines for the transition process and posting them on the walls of clinics for all doctors } \\
\text { and patients to see }\end{array}$ & 88 & 93 \\
Having a social worker/trained volunteer assist in transition from start to finish by attending all appointments & 93 & 68 \\
with the adolescent patient & 90 & 90 \\
Providing sexual health-related counselling at all appointments & 100 & 88 \\
Organising support group meetings for adolescents in transition & 98 \\
Providing comprehensive services at all appointments, including primary care, dental care and on-site pharmacy services & 98 & 80 \\
Providing help with transportation and medical costs & 93 & 89 \\
Introducing the new adult provider when adolescents are still at the youth clinic & 93 & 80 \\
Having a team meeting between adolescent and adult providers to do handover of patient history & 93 & 82 \\
Providing follow-up appointments with the paediatric provider after the transition to the adult clinic & 75 & 71 \\
Beginning transition to adult care at age 18 years & 70 & 73 \\
Beginning transition to adult care at age 24 years & 30 & 45
\end{tabular}


Table 4. Provider recommendations for improved healthcare transition in SA

\begin{tabular}{|c|c|}
\hline Proposal & Justification \\
\hline $\begin{array}{l}\text { Eliminate age as an absolute } \\
\text { indicator for transition }\end{array}$ & $\begin{array}{l}\text { While the patient should be at least } 18 \text { years old, maturity and life stage are more indicative of transition readiness. } \\
\text { Providers should consider other factors including: finished high school, attends appointments alone, been through } \\
\text { the African cultural manhood initiation ritual (males), requests to be transferred out, and has stable health. }\end{array}$ \\
\hline $\begin{array}{l}\text { Inform patients of transition } \\
\text { early on in adolescent care }\end{array}$ & Prior patient knowledge of impending transition will reduce the shock of the transfer. \\
\hline $\begin{array}{l}\text { Improve communication } \\
\text { between paediatric and adult } \\
\text { care providers }\end{array}$ & $\begin{array}{l}\text { Healthcare providers want to send their patients to a known clinic and known doctor. Adolescent clinics can } \\
\text { begin developing relationships with specific adult clinics. Creating a physical, formalised card for adolescent } \\
\text { patients to bring to adult clinics may ensure the adult doctor receives the referral letter. This physical card will } \\
\text { contain all relevant medical, social, and educational information. }\end{array}$ \\
\hline $\begin{array}{l}\text { Create standardised } \\
\text { guidelines for the transition } \\
\text { process }\end{array}$ & $\begin{array}{l}\text { A validated standard will bolster provider confidence in determining when patients are ready to transition. } \\
\text { Patients will also have informed expectations. We recommend adolescent healthcare provider involvement in the } \\
\text { creation of these guidelines. }\end{array}$ \\
\hline $\begin{array}{l}\text { Create support groups for } \\
\text { transition-age adolescents }\end{array}$ & $\begin{array}{l}\text { Transition-age adolescents can meet to discuss disclosure, stigma and other issues surrounding the transition. } \\
\text { The support groups may address entering both adult care and adulthood. All interviewees strongly agree this } \\
\text { intervention would be feasible and helpful. Adolescents can 'graduate' together from these support groups and } \\
\text { move to adult clinics in familiar peer groups. }\end{array}$ \\
\hline $\begin{array}{l}\text { Train adult staff in providing } \\
\text { youth-friendly care }\end{array}$ & $\begin{array}{l}\text { Workshops can teach adult staff to treat younger patients in a respectful and non-judgemental manner. We } \\
\text { recommend adolescent healthcare provider involvement in this training process. }\end{array}$ \\
\hline $\begin{array}{l}\text { Transfer stable youth into } \\
\text { adult ART adherence clubs }\end{array}$ & $\begin{array}{l}\text { In an ART adherence club, } 30 \text { patients meet at a community venue for } 1 \text { hour every } 2 \text { months. Group meetings } \\
\text { are facilitated by a lay club facilitator who provides a quick clinical assessment and dispenses prepacked ART. } \\
\text { These clubs allow for consistency in seeing the same nurse, counsellor, and fellow club members, as well as } \\
\text { convenience with a school or work schedule. }\end{array}$ \\
\hline
\end{tabular}

nearly all SA healthcare staff are overburdened, it appears paediatric healthcare providers have a little more time - and motivation - to take the lead in facilitating the transition.

\section{Study limitations}

Several limitations affected our research. Most significant was the small sample size in both arms. Accordingly, our results may not be generalisable to all SA healthcare providers. Additionally, the majority of interviewees served predominantly perinatally infected adolescents, so our results may differ from the perspectives of healthcare providers catering to behaviourally infected youth. Both survey and interview portions of the study were probably affected by selection bias, as recruitment for both arms was achieved through convenience sampling.

\section{Conclusion}

Despite the above limitations, we believe these insights warrant further investigation. Increasing numbers of SA children living with HIV are maturing into adolescence and into adulthood, yet current outcomes among HIV-positive adolescents are universally poor. More research in management of these adolescents including better models for healthcare transition is urgent.

Acknowledgements. The authors would like to thank ViiV Healthcare for their support of the Desmond Tutu HIV Foundation's Youthshield Project. The authors would also like to thank the Hanan-CRUSAID clinic, counsellors and staff for their dedication and work. THK would like to thank Dr Gene T Richardson for his mentorship.

Disclosure statement. THK was funded by an Omidyar Network Fellowship through the Stanford University Haas Center for Public
Service. The funders had no role in study design, data collection and analysis, decision to publish, or preparation of the manuscript.

\section{References}

1. Ferrand RA, Corbett EL, Wood R, et al. AIDS among older children and adolescents in southern Africa: Projecting the time course and magnitude of the epidemic. AIDS 2009;23(15):2039-2046. Africa: Projecting the time course and
DOI:10.1097/QAD.0b013e32833016ce

2. Gilliam PP, Ellen JM, Leonard L, et al. Transition of adolescents with HIV to adult care: Characteristics . Gilliam PP, Ellen JM, Leonard L, et al. Transition of adolescents with HIV to adult care: Characteristics
and current practices of the adolescent trials network for HIV/AIDS interventions. J Assoc Nurses

AIDS Care 2011;22(4):283-294. DOI:10.1016/j.jana.2010.04.003

Viner RM. Transition of care from paediatric to adult services: One part of improved health services for adolescents. Arch Dis Child 2008;93(2):160-163. DOI:10.1136/adc.2006.103721

4. Vijayan T, Benin AL, Wagner K, et al. We never thought this would happen: Transitioning care of adolescents with perinatally acquired HIV infection from pediatrics to internal medicine. AIDS Care 2009;21(10):1222-1229. DOI:10.1080/09540120902730054

5. Machado DM, Succi RC, Turato ER. Transitioning adolescents living with HIV/AIDS to adult-oriented health care: An emerging challenge. J Pediatr 2010;86(6):465-472. DOI:10.2223/jped.2048

6. Maturo D, Powell A, Major-Wilson $\mathrm{H}$, et al. Development of a protocol for transitioning adolescents with HIV infection to adult care. J Pediatr Health Care 2011;25(1):16-23. DOI:10.1016/j. pedhc.2009.12.005

7. Jaspan $\mathrm{HB}$, Flisher $\mathrm{AJ}$, Myer L, et al. Sexual health, HIV risk, and retention in an adolescent HIV-prevention trial preparatory cohort. J Adolesc Health 2011;49(1):42-46. DOI:10.1016/j. jadohealth.2010.10.009

8. Blum RW. Youth in sub-Saharan Africa. J Adolesc Health 2007;41(3):230-238. DOI:10.1016/j.jadohealth.2007.04.005

9. Ritchie J, Lewis J, Nicholls CM, et al. Qualitative Research Practice: A Guide for Social Science Students and Researchers. London: Sage, 2013

10. Katusiime C, Parkes-Ratanshi R, Kambugu A. Transitioning behaviourally infected HIV-positive young people into adult care: Experiences from the young person's point of view. South Afr J HIV Med 2013;14(1):20,22-24. DOI:10.7196/sajhivmed.885

1. Hallgren KA. Computing inter-rater reliability for observational data: An overview and tutorial. Tutor Quant Methods Psychol 2012;8(1):23.

2. Matthew B, Miles A, Huberman M. Qualitative Data Analysis: An Expanded Sourcebook. Thousand Oaks, CA: Sage, 1994.

13. Reiss JG, Gibson RW, Walker LR. Health care transition: Youth, family, and provider perspectives. Pediatrics 2005;115(1):112-120.

14. Monin JK, Schulz R, Kershaw TS. Caregiving spouses' attachment orientations and the physical and psychological health of individuals with Alzheimer's disease. Aging Ment Health 2013;17(4):508-516. DOI:10.1080/13607863.2012.747080

15. Ciechanowski PS, Katon WJ, Russo JE, Walker EA. The patient-provider relationship: Attachment theory and adherence to treatment in diabetes. Am J Psychiatry 2001;158(1):29-35. 